\title{
A Stabilized Formulation for Incompressible Plasticity Using Linear Triangles and Tetrahedra
}

\author{
M. Chiumenti*, Q. Valverde', C. Agelet de Saracibar* \\ and M. Cervera* \\ * International Center for Numerical Methods in Engineering (CIMNE) \\ Universidad Politécnica de Cataluña (UPC) \\ Edificio C1, Campus Norte, Gran Capitán s/n, 08034 Barcelona Spain. \\ ${ }^{+}$Departamento de Ingeniería - Sección Ingeniería Mecánica \\ Pontificia Universidad Católica del Perú, Lima, Perú.
}

KEYWORDS: incompressibility, plasticity, orthogonal sub-grid scales, stabilized finite element method

\begin{abstract}
In this paper a stabilized finite element method to deal with incompressibility in solid mechanics is presented. Both elastic and J2-plastic constitutive behaviour have been considered. A mixed formulation involving pressure and displacement fields is used and a continuous linear interpolation is considered for both fields. To circumvent the Babuška-Brezzi condition a stabilization technique based on the orthogonal sub-scale method is introduced. The main advantage of the method is the possibility of using linear triangular or tetrahedral finite elements, which are easy to generate for real industrial applications. Results are compared with standard Galerkin and Q1P0 mixed formulations in either elastic or elasto-plastic incompressible problems.
\end{abstract}




\section{Introduction}

This paper proposes a possible solution to the problem of incompressibility observed in solid mechanics in case of either elastic incompressibility or J2 plasticity. The objective is to avoid the so called volumetric locking, an undesirable effect exhibited by all low order elements based on the standard Galerkin formulation. Many successful strategies to avoid volumetric locking based on both mixed and enhanced formulations can be found in the literature, Miehe (1994), Simo et al. (1990), Simo et al. (1985), but they generally fail in the case of linear triangular or tetrahedral elements due to the lack of satisfaction of the Babuška-Brezzi condition (Brezzi (1991)). Other formulations have been proposed by Zienkiewicz et al. (1998), Taylor (1999), Oñate et al. (2002), Klass et al. (1999a-1999b), etc. to deal with such elements, mainly motivated by the fact that nowadays, for real life geometries, tetrahedral meshes are relatively easy to generate. Techniques based on the sub-grid scale approach proposed by Hughes (1995), have been applied in the context of solid mechanics in strain localization problems by Garikipati et

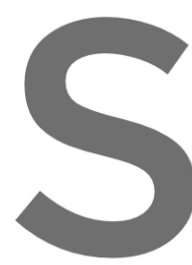
al. (1998) and Garikipati et al. (200
seale is represented by additional ad-
the same fashion than in the assume
based on the orthogonal sub-scales
has been applied to incompressible
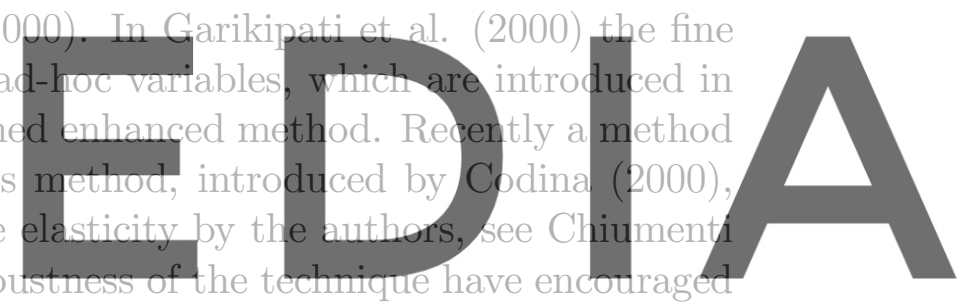

the authors to extend the approach to non-linear problems. An equal order

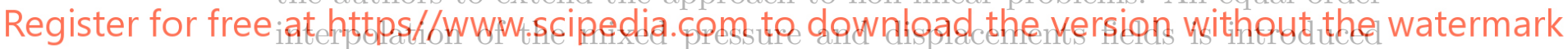

followed by a decomposition of the unknowns into resolvable and sub-grid

scales orthogonal to the finite element space. The basic idea is to approximate the effect of the component of the continuous solution which cannot be captured by the finite element solution and is the cause of the volumetric locking.

In the next section the equations that define the mechanical problem including the condition of incompressibility will be presented. Later on, the stabilization model using the orthogonal sub-grid scale approach will be presented taking into account the non-linear behavior induced by the plastic model. Finally, some numerical benchmarks will compare the standard Galerkin formulation as well as the mixed Q1P0 approach with the present formulation. 


\section{Mixed formulation for J2 plasticity model}

It is well known that elements coming from standard displacement formulation often lock in constrained media problems, such as incompressible problems. A possible alternative can be stated introducing a mixed variational formulation. Within this framework it is possible to design more robust and flexible elements such as the assumed stress elements where pressure is interpolated independently of the displacement field.

In this section a mixed formulation to deal with an elasto-plastic behavior is introduced. The finite element discretization is presented to point out the restrictions of this formulation coming from the Babuška-Brezzi stability condition. Furthermore, the sub-grid scale approach is presented as an alternative to circumvent this condition to be able to use continuous linear interpolations for both displacements and pressure fields. Finally, the stabilized system of equations that solve the problem of incompressibility is presented, assuming as a particular choice the orthogonal sub-grid scales.
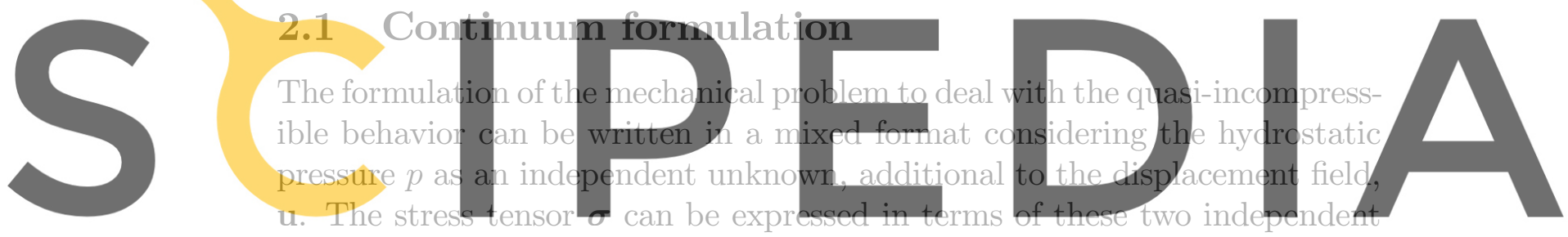

variables such as:

Register for free at https//www.scipedia.com to download the version without the watermark

$$
\sigma(p, \mathbf{u})=p \mathbb{1}+\mathbf{s}(\mathbf{u}) \quad\left\{\begin{array}{l}
p=\frac{1}{3} \operatorname{tr}(\sigma) \\
\mathbf{s}=\operatorname{dev}(\sigma)
\end{array}\right.
$$

where $p$ and $\mathbf{s}(\mathbf{u})$ are the volumetric and the deviatoric parts of the stress tensor, respectively.

If we refer to J2 plasticity then the volumetric and the deviatoric constitutive behavior can be split and treated independently. In particular, the hydrostatic pressure $p$ can be expressed as:

$$
p=K \varepsilon_{v}
$$

where $K$ is the bulk modulus, also referred to as modulus of volumetric compressibility and $\varepsilon_{v}=\operatorname{tr}(\varepsilon)=\nabla \cdot \mathbf{u}$ is the volumetric part of the total 
deformation $\varepsilon=\nabla^{s} \mathbf{u}$. On the other hand, the deviatoric part of the stress tensor $\mathbf{s}$ can be introduced as:

$$
\mathbf{s}=2 G \mathbf{e}^{e}=2 G\left(\mathbf{e}-\mathbf{e}^{p}\right)
$$

where $G$ is the shear modulus and $\mathbf{e}^{e}$ and $\mathbf{e}$ are the deviatoric components of the elastic and the total strain tensors, respectively. According to J2-plastic model, plastic deformation, $\mathbf{e}^{p}$, is assumed to be only deviatoric and it has been computed according to the J2-plastic constitutive model summarized in Box-1 (see Agelet de Saracibar et al. 2001).

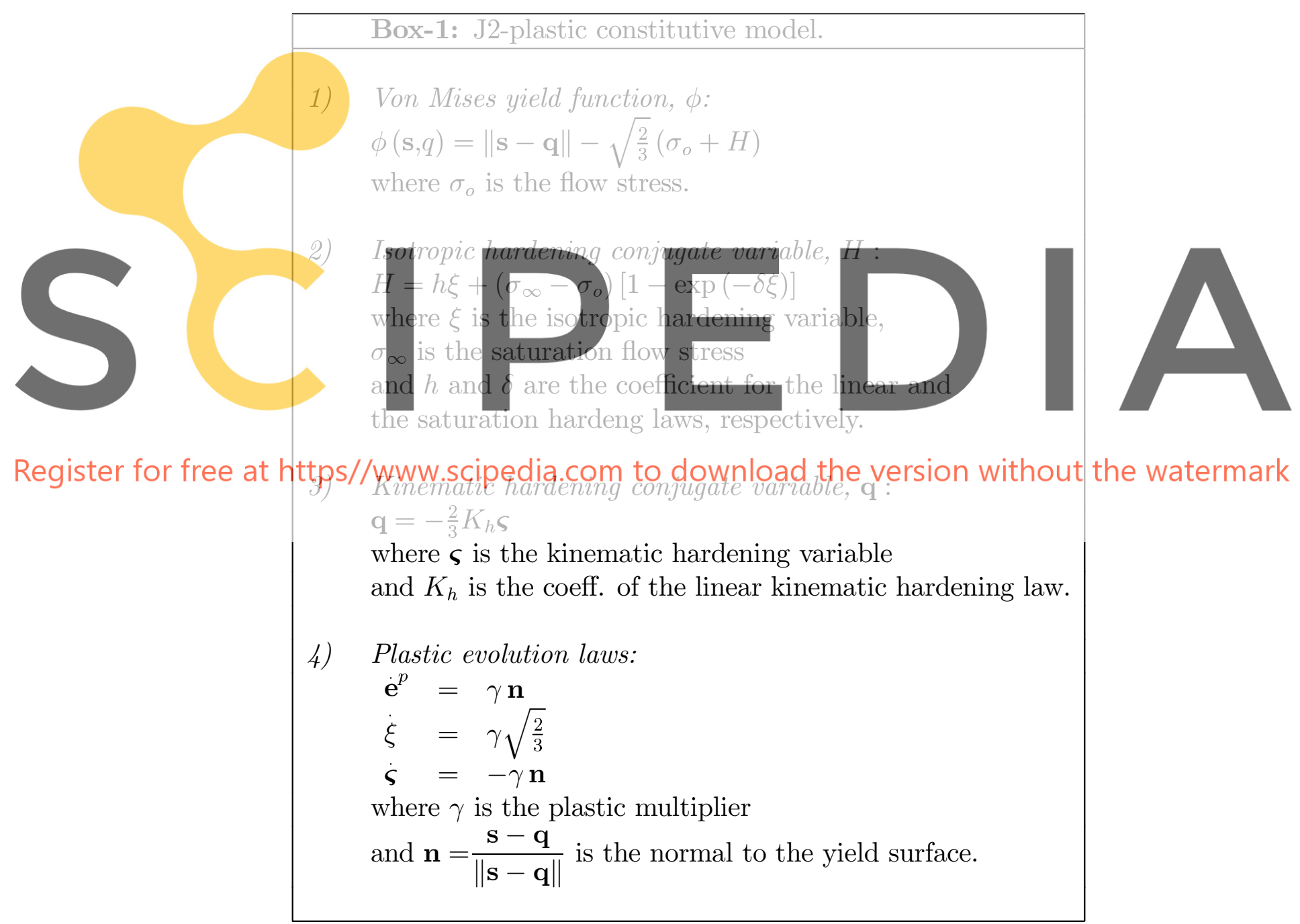


Therefore, the strong form of the mixed problem that we want to solve, can be formulated as: find the displacement field $\mathbf{u}$ and the pressure field $p$, for given prescribed body forces $\mathbf{f}$, such that:

$$
\begin{array}{rll}
\nabla \cdot \mathbf{s}+\nabla p+\mathbf{f} & =\mathbf{0} & \text { in } \Omega \\
\nabla \cdot \mathbf{u}-\frac{1}{K} p=0 & \text { in } \Omega
\end{array}
$$

where $\Omega$ stands for an open and bounded domain of $\mathbb{R}^{n_{\mathrm{dim}}}$ in a space of $n_{\mathrm{dim}}$ dimensions. Equations (4)-(5) are subjected to appropriate Diritchlet and Neumann boundary conditions in terms of prescribed displacements, $\mathbf{u}=\overline{\mathbf{u}}$ on $\partial \Omega_{u}$, and prescribed tractions, $\bar{t}=\sigma \cdot \mathrm{n}$ on $\partial \Omega_{t}$, respectively.

Note that the formulation is valid for both compressible and incompressible cases. In particular, in case of incompressibility, that is when $K \rightarrow \infty$, equation (5), transforms into:

$$
\nabla \cdot \mathbf{u}=0 \quad \text { in } \Omega
$$

Denoting by $/$ the inner product in the space of sculare interroble

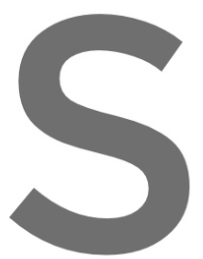

functions $L$ stated as:
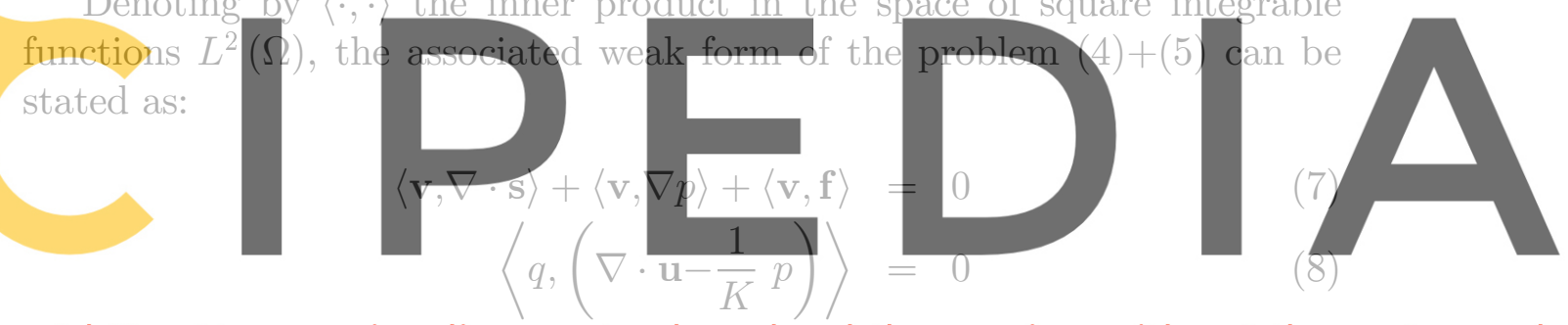

Register for free at https//www.scipedia,com to download the version without the watermark where $\mathrm{V}$ and $q$ are the variations of the displacements and pressure fields,

respectively.

Integrating by parts, problem (7-8) can be rewritten as

$$
\begin{aligned}
\left\langle\nabla^{s} \mathbf{v}, \mathbf{s}\right\rangle+\langle\nabla \cdot \mathbf{v}, p\rangle-\langle\mathbf{v}, \mathbf{f}\rangle-\langle\mathbf{v}, \overline{\mathbf{t}}\rangle_{\partial \Omega_{t}} & =0 \\
\left\langle q,\left(\nabla \cdot \mathbf{u}-\frac{1}{K} p\right)\right\rangle & =0
\end{aligned}
$$

Observe that (9-10) involve the first derivative of the displacements and only the primal function of the pressure field. Hence, the natural spaces for displacements and pressure continuum fields are: $\mathbf{u} \in \mathcal{V}$ and $p \in \mathcal{Q}$ where $\mathcal{V}=H^{1}(\Omega)$ where $\mathcal{V}=H^{1}(\Omega)$ and $\mathcal{Q}=L^{2}(\Omega)$, respectively. The corresponding variations are defined in: $\mathbf{v} \in \mathcal{V}_{0}=\left\{\mathbf{v} \in \mathcal{V} \mid \mathbf{v}=\mathbf{0}\right.$ en $\left.\partial \Omega_{u}\right\}$ and 
$q \in \mathcal{Q} . H^{m}(\Omega)$ denotes the space of functions of which its derivatives up to order $m \geq 0$ (integer) belong to $L^{2}(\Omega)$. Roughly speaking, $H^{1}(\Omega)$ involves continuous functions with discontinuous derivatives, while $L^{2}(\Omega)$ includes even discontinuous functions.

Using a compact notation, the above problem can be stated as the following bilinear form :

$$
R(\mathbf{U}, \mathbf{V})=\mathbf{0} \quad \forall \mathbf{V}
$$

where $\mathbf{U}:=[\mathbf{u}, p]^{t} \in \mathcal{W}=\mathcal{V} \times \mathcal{Q}$ and $\mathbf{V}:=[\mathbf{v}, q]^{t} \in \mathcal{W}_{0}=\mathcal{V}_{0} \times \mathcal{Q}$ are the vector of unknowns and its variation, respectively.

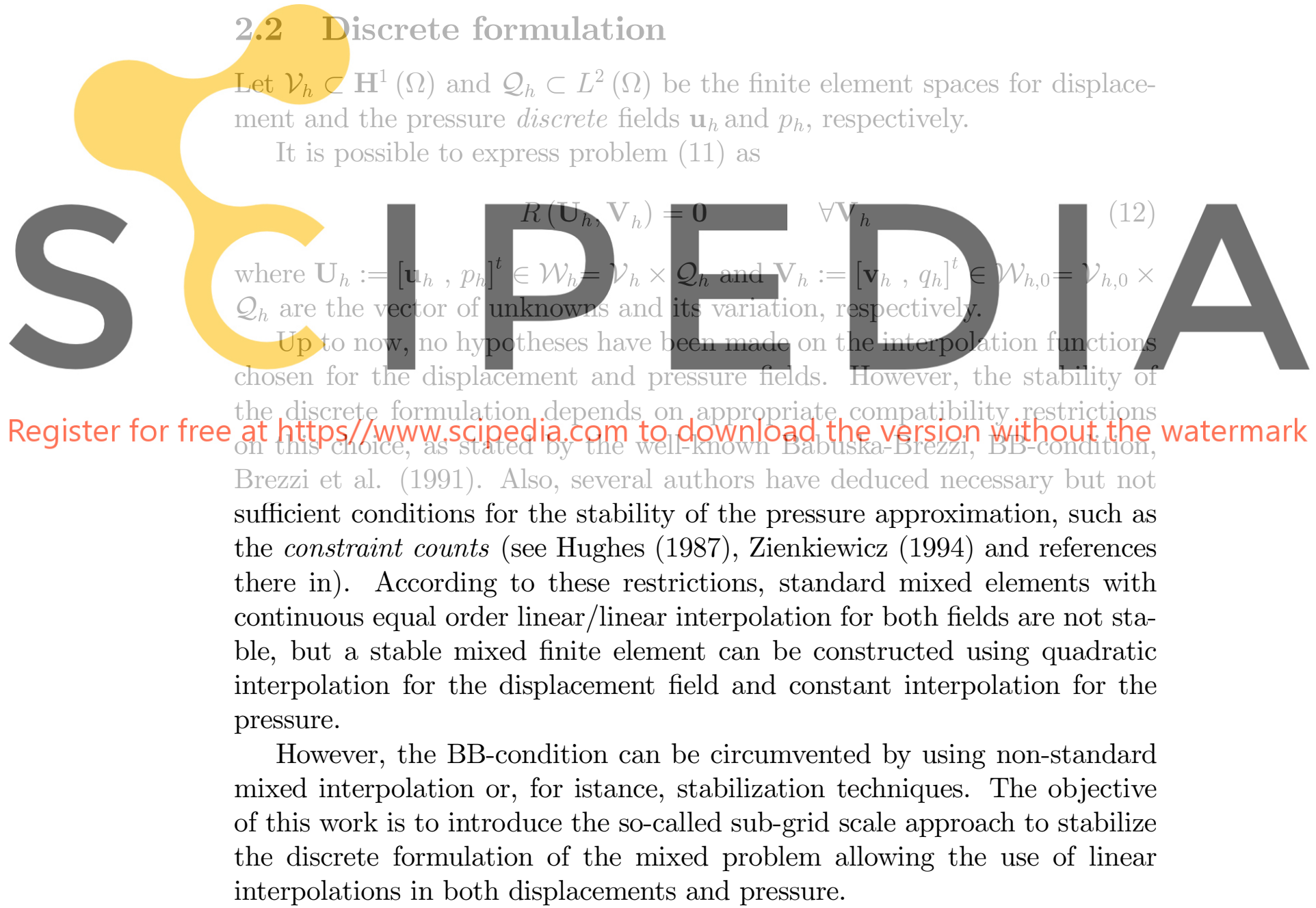




\subsection{The sub-grid scale approach}

The basic idea of the sub-grid scale approach (Hughes (1995)) is to consider that the continuous displacement field can be approximated considering two components, one coarse and a finer one, corresponding to different scales or levels of resolution. The solution of the continuous problem contains components from both scales. For the solution of the discrete problem to be stable it is necessary to, somehow, include the effect of both scales in the approximation. The coarse scale can be appropriately solved by a standard finite element interpolation, which however cannot solve the finer scale. Nevertheless, the effect of this finer scale can be included, at least locally, to enhance the stability of the pressure in the mixed formulation.

Therefore, the solution of the mixed problem will be approximated as

$$
\mathrm{U}=\mathrm{U}_{h}+\widetilde{\mathrm{U}}
$$

\section{that is}
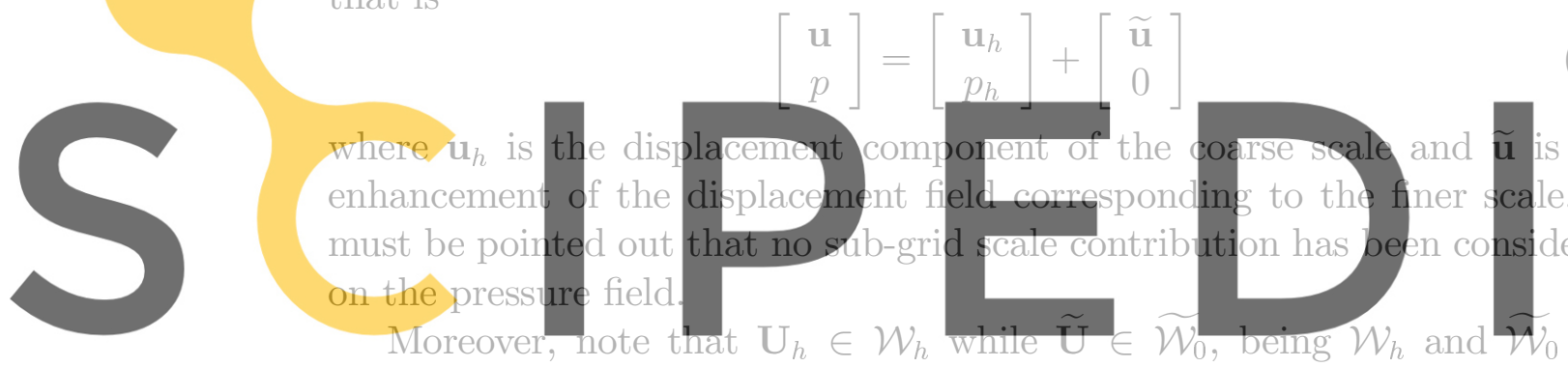

finite element and the sub-grid scale space, respectively. It is important to

Register for free at https//www.scipedia.com to download the versjon without the watermark

the subgrid scale displacements vanishes all over the boundary $\partial \Omega$. Hence,

the solution $\mathbf{U}$ belongs to the refined space:

$$
\mathcal{W}=\mathcal{W}_{h} \oplus \widetilde{\mathcal{W}_{0}}
$$

Assuming an additive split of the total deviatoric strain tensor $\mathbf{e}=\mathbf{e}^{e}+\mathbf{e}^{p}$, it is reasonable to assume the elastic and plastic components as:

$$
\begin{aligned}
\mathbf{e}^{e} & =\mathbf{e}_{h}^{e}+\widetilde{\mathbf{e}}^{e} \\
\mathbf{e}^{p} & =\mathbf{e}_{h}^{p}
\end{aligned}
$$

Observe that this supposes that $\widetilde{\mathbf{u}}$ is sufficiently small, compared to the coarse solution $\mathbf{u}_{h}$, so that the deformations induced by the sub-grid displacements $\widetilde{\mathbf{u}}$ are purely elastic, that is $\widetilde{\mathbf{e}}^{e}=\widetilde{\mathbf{e}}$. This is arguable considering 
$\widetilde{\mathbf{u}}$ as a perturbation of the finite element field $\mathbf{u}_{h}$, which cannot be resolved in $\mathcal{V}_{h}$. As a consequence, the deviatoric stress tensor $\mathbf{s}\left(\mathbf{u}_{h}+\widetilde{\mathbf{u}}\right)$ can be split as the sum of a first contribution $\mathbf{s}_{h}\left(\mathbf{u}_{h}\right)$, coming from the elasto-plastic behavior induced by the coarse solution $\mathbf{u}_{h}$, and a second contribution $\widetilde{\mathbf{s}}(\widetilde{\mathbf{u}})$, associated with the sub-grid scale $\widetilde{\mathbf{u}}$, in the form:

$$
\mathbf{s}\left(\mathbf{u}_{h}+\widetilde{\mathbf{u}}\right)=\mathbf{s}_{h}\left(\mathbf{u}_{h}\right)+\widetilde{\mathbf{s}}(\widetilde{\mathbf{u}})
$$

so that:

$$
\begin{aligned}
\mathbf{s}_{h}\left(\mathbf{u}_{h}\right) & =2 G \mathbf{e}_{h}^{e}=2 G\left(\mathbf{e}_{h}-\mathbf{e}_{h}^{p}\right) \\
\widetilde{\mathrm{s}}(\widetilde{\mathbf{u}}) & =2 G \widetilde{\mathrm{e}}^{e} \cong 2 G \widetilde{\mathrm{e}}
\end{aligned}
$$

In this way, the continuous problem (11) transforms into finding $\mathbf{U}_{h}$ and U such that:

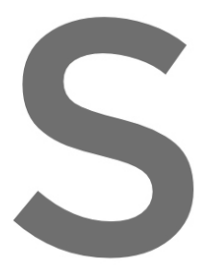

$$
R\left(\mathrm{U}, \mathbf{V}_{h}\right)=R\left(\mathrm{U}_{h}+\widetilde{\mathrm{U}}, \mathbf{V}_{h}\right)=0 \quad \forall \mathbb{V}_{h} \in \mathcal{W}_{h}
$$

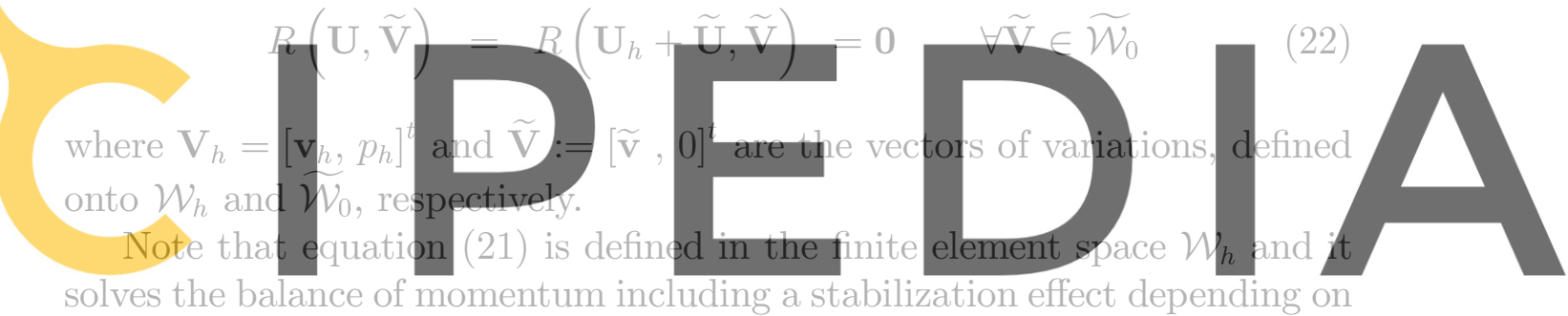

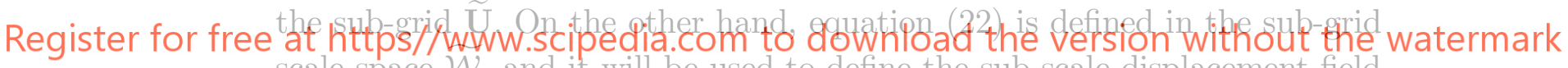
scale space $\mathcal{W}_{0}$ and it will be used to define the sub-scale displacement field $\widetilde{\mathrm{U}}$.

Particularizing problem (21) together with the above assumption, it follows that:

$$
\begin{array}{r}
\left\langle\mathbf{v}_{h}, \nabla \cdot \mathbf{s}\right\rangle+\left\langle\mathbf{v}_{h}, \nabla p_{h}\right\rangle+\left\langle\mathbf{v}_{h}, \mathbf{f}\right\rangle=0 \\
\left\langle q_{h},\left(\nabla \cdot\left(\mathbf{u}_{h}+\widetilde{\mathbf{u}}\right)-\frac{1}{K} p_{h}\right)\right\rangle=0
\end{array}
$$

Integrating by parts, problem (23) can be rewritten as:

$$
\begin{aligned}
\left\langle\nabla^{s} \mathbf{v}_{h}, \mathbf{s}\right\rangle+\left\langle\nabla \cdot \mathbf{v}_{h}, p_{h}\right\rangle-\left\langle\mathbf{v}_{h}, \mathbf{f}\right\rangle-\left\langle\mathbf{v}_{h}, \overline{\mathbf{t}}\right\rangle_{\partial \Omega_{t}} & =0 \\
\left\langle q_{h},\left(\nabla \cdot\left(\mathbf{u}_{h}+\widetilde{\mathbf{u}}\right)-\frac{1}{K} p_{h}\right)\right\rangle & =0
\end{aligned}
$$


where the integral over the boundary $\partial \Omega_{t}$ involves the prescribed traction $\overline{\mathbf{t}}$. Finally, expressing the total deviatoric stress tensor $\mathbf{s}$ in terms of $\mathbf{s}_{h}$, we obtain:

$$
\begin{aligned}
\left\langle\nabla^{s} \mathbf{v}_{h}, \mathbf{s}_{h}\right\rangle+\left\langle\nabla^{s} \mathbf{v}_{h}, \widetilde{\mathbf{s}}\right\rangle+\left\langle\nabla \cdot \mathbf{v}_{h}, p_{h}\right\rangle-\left\langle\mathbf{v}_{h}, \mathbf{f}\right\rangle-\left\langle\mathbf{v}_{h}, \overline{\mathbf{t}}\right\rangle_{\partial \Omega_{t}} & =0 \\
\left\langle q_{h},\left(\nabla \cdot\left(\mathbf{u}_{h}+\widetilde{\mathbf{u}}\right)-\frac{1}{K} p_{h}\right)\right\rangle & =0
\end{aligned}
$$

Note that it is possible to split these equations in the same way as for the purely elastic case (see Chiumenti et al. (2002)), so that:

$$
R\left(\mathbb{U}, \mathbb{V}_{h}\right)=R\left(\mathbb{U}_{h}, \mathbb{V}_{h}\right)+R\left(\widetilde{\mathbf{U}}, \mathbb{V}_{h}\right)=0 \quad \forall \mathbb{V}_{h} \in \mathcal{W}_{h}
$$

where $R\left(\mathbf{U}_{h}, \mathbf{V}_{h}\right)$ is the standard term of the weak form of the problem

$$
R\left(\mathbf{U}_{h}, \mathbf{V}_{h}\right)=\left[\begin{array}{c}
\left\langle\nabla^{s} \mathbf{v}_{h}, \mathbf{s}_{h}\right\rangle+\left\langle\nabla \cdot \mathbf{v}_{h}, p_{h}\right\rangle-\left\langle\mathbf{v}_{h}, \mathbf{f}\right\rangle-\left\langle\mathbf{v}_{h}, \overline{\mathbf{t}}\right\rangle_{\partial \Omega_{t}} \\
\left\langle q_{h}, \nabla \cdot \mathbf{u}_{h}\right\rangle-\left\langle q_{h}, \frac{1}{K} p_{h}\right\rangle
\end{array}\right]
$$
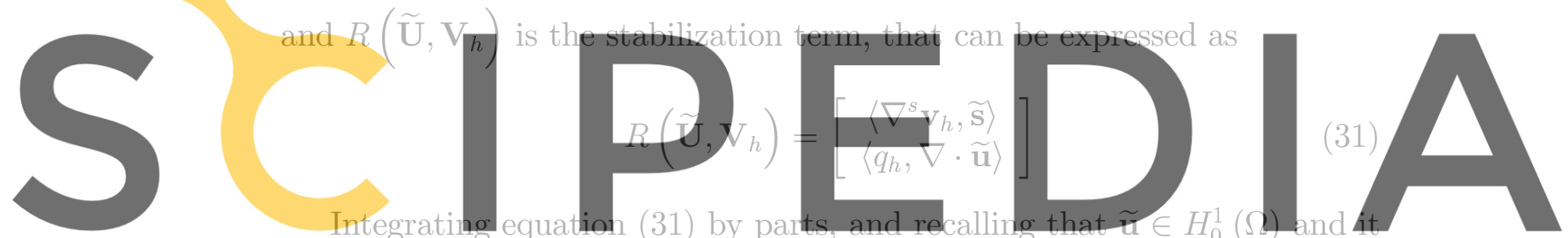

vanishes all over the boundary $\partial \Omega$, the result is

Register for free at https//www.scipedia.com to download the version without the watermark

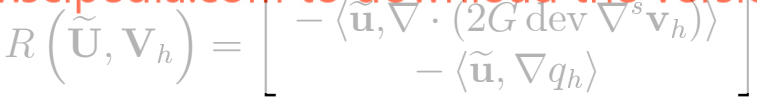

The pending subject for the next section is how to approximate the subscale displacements $\widetilde{\mathbf{u}}$ to define it properly.

\subsection{Orthogonal sub-grid scales}

The objective in this section is to obtain a useful expression for the sub-grid scale displacements $\widetilde{\mathbf{u}}$ to be introduced in (32). To achieve this result, let us manipulate problem (22), introducing the split of the stress tensor (18), in the form:

$$
R(\mathbf{U}, \widetilde{\mathbf{V}})=\left[\begin{array}{c}
\left\langle\widetilde{\mathbf{v}}, \nabla \cdot \mathbf{s}_{h}\right\rangle+\langle\widetilde{\mathbf{v}}, \nabla \cdot \widetilde{\mathbf{s}}\rangle+\left\langle\widetilde{\mathbf{v}}, \nabla p_{h}\right\rangle+\langle\widetilde{\mathbf{v}}, \mathbf{f}\rangle \\
0
\end{array}\right]=\mathbf{0}
$$


where the second equation vanishes due to the fact that $\widetilde{p}=0$ (see eq. 14). Observe that also in this case it is possible to split the problem into:

$$
R\left(\mathbf{U}_{h}, \widetilde{\mathbf{V}}\right)+R(\widetilde{\mathbf{U}}, \widetilde{\mathbf{V}})=\mathbf{0} \quad \forall \widetilde{\mathbf{V}} \in \widetilde{\mathcal{W}}_{0}
$$

where the first term only depends on $\mathbf{U}_{h}$, while the second term includes the effect of $\widetilde{\mathbf{U}}$. The above equation can be rewritten as:

$$
\langle\widetilde{\mathbf{v}}, \nabla \cdot \widetilde{\mathbf{s}}(\widetilde{\mathbf{u}})\rangle+\left\langle\widetilde{\mathbf{v}}, \mathbf{r}_{h}\right\rangle=0
$$

where the following operator has been introduced:

$$
\mathbf{r}_{h}=\nabla \cdot \mathbf{s}_{h}+\nabla p_{h}+\mathbf{f}
$$

Having in mind the expression of the deviatoric part of the stress tensor

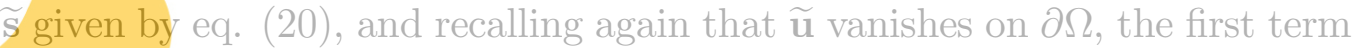
in (35) can be developed as:

$$
\langle\widetilde{\mathbf{v}}, \nabla \cdot \widetilde{\mathbf{s}}(\widetilde{\mathbf{u}})\rangle=-\left\langle\nabla^{s} \widetilde{\mathbf{v}}, 2 G \operatorname{dev} \nabla^{s} \widetilde{\mathbf{u}}\right\rangle
$$

Taking into account that for a given a function $(\cdot)$ the order of its gradient
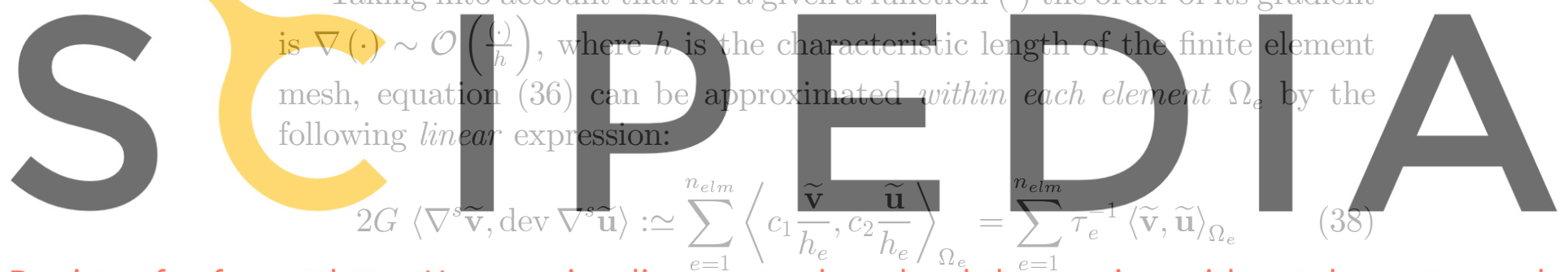

Register for free at https//www.scipedia.com to download the version without the watermark

where $\tau_{e}=\frac{c h_{e}^{2}}{2 G}$ is a parameter defined as a function of the characteristic

length of the element $h_{e}$ and the shear modulus $G$; coefficient $c=c_{1} \cdot c_{2}$ is a coefficient depending on the element interpolation, to be appropriately chosen.

Substituting the above results in (35) then:

$$
\sum_{e=1}^{n_{e l m}}\langle\widetilde{\mathbf{v}}, \widetilde{\mathbf{u}}\rangle_{\Omega_{e}}=\sum_{e=1}^{n_{e l m}} \tau_{e}\left\langle\widetilde{\mathbf{v}}, \mathbf{r}_{h}\right\rangle_{\Omega_{e}}
$$

Moreover, as a particularization of the general form of the sub-grid scale method detailed in the previous section, let us choose as sub-grid scale space $\widetilde{\mathcal{W}}_{0}$, the space orthogonal to the standard finite element space $\mathcal{W}_{h, 0}$, that is:

$$
\widetilde{\mathcal{W}_{0}} \approx \mathcal{W}_{h, 0}^{\perp}
$$


The space $\mathcal{W}_{h, 0}^{\perp}$ is generally referred as the space of orthogonal sub-scales. It was introduced in Codina (2000), and applied to incompressible elasticity by Chiumenti et al. (2002).

Imposing that $\widetilde{\mathbf{u}} \in \mathcal{W}_{h, 0}^{\perp}$, the following approximation can be established:

$$
\sum_{e=1}^{n_{e l m}}\langle\widetilde{\mathbf{v}}, \widetilde{\mathbf{u}}\rangle_{\Omega_{e}}=\sum_{e=1}^{n_{e l m}} \tau_{e}\left\langle\widetilde{\mathbf{v}}, P_{h}^{\perp}\left(\mathbf{r}_{h}\right)\right\rangle_{\Omega_{e}} \quad \forall \widetilde{\mathbf{v}} \in \mathcal{W}_{h, 0}^{\perp}
$$

where $P_{h}^{\perp}$ is the orthogonal projection onto $\mathcal{W}_{h}^{\perp}$.

It must be pointed out that $\mathbf{f}$ in (36) belongs to the space $\mathcal{W}_{h}$ and therefore $P_{h}^{\perp}(\mathbf{f})=\mathbf{0}$. On the other hand, $\nabla \cdot \mathbf{s}_{h}$ also in (36), involves second derivatives of finite element functions which vanish when linear elements are used. As a result

$$
\sum_{e=1}^{n_{e l m}}\langle\widetilde{\mathbf{v}}, \widetilde{\mathbf{u}}\rangle_{\Omega_{e}}=\sum_{e=1}^{n_{\text {elm }}} \tau_{e}\left\langle\widetilde{\mathbf{v}}, P_{h}^{\perp}\left(\nabla p_{h}\right)\right\rangle_{\Omega_{e}} \quad \forall \widetilde{\mathbf{v}} \in \mathcal{W}_{h, 0}^{\perp}
$$

Thus, the sub-grid scale displacements $\widetilde{\mathbf{u}}$ can be approximated within each element $\Omega_{e}$, as:

$$
\widetilde{\mathbf{u}}_{e} \simeq \tau_{e}\left[\nabla p_{h}-P_{h}\left(\nabla p_{h}\right)\right]
$$

where the orthogonal projection $P_{h}^{\perp}$ of a variable $(\cdot)$ has been computed as:

$$
P_{h}^{\perp}(\cdot)=(\cdot)-P_{h}(\cdot)
$$

where $P_{h}(\cdot)$ is the projection onto the finite element space $\mathcal{V}_{h}$, computed as:

$$
\left\langle P_{h}(\cdot), \boldsymbol{\eta}_{h}\right\rangle=\left\langle(\cdot), \boldsymbol{\eta}_{h}\right\rangle \quad \forall \boldsymbol{\eta}_{h} \in \mathcal{V}_{h}
$$

In particular, calling $\boldsymbol{\Pi}_{h}=P_{h}\left(\nabla p_{h}\right)$ the projection onto $\mathcal{V}_{h}$ of the pressure gradient, it can been computed as:

$$
\left\langle\boldsymbol{\Pi}_{h}, \boldsymbol{\eta}_{h}\right\rangle=\left\langle\nabla p_{h}, \boldsymbol{\eta}_{h}\right\rangle \quad \forall \boldsymbol{\eta}_{h} \in \mathcal{V}_{h}
$$

leading to a final expression for the approximation of the sub-grid scale, within each element, such as:

$$
\widetilde{\mathbf{u}}_{e} \simeq \tau_{e}\left[\nabla p_{h}-\boldsymbol{\Pi}_{h}\right]
$$

Observe that, according to the objective of this work, the use of elementwise linear interpolation for the pressure, implies a discontinuous field for the pressure gradient and, consequently, for the sub-grid scale solution $\widetilde{\mathbf{u}}_{e}$. On 
the other hand, $\Pi_{h}$ is defined as a continuous field, leading to an expression for $\widetilde{\mathbf{u}}_{e}$ which cannot be condensed element by element.

Substituting the approximation of $\widetilde{\mathbf{u}}_{e}$ into the expression of the stabilization term (32) then it simplifies as

$$
R\left(\widetilde{\mathbf{U}}, \mathbf{V}_{h}\right)=\left[\begin{array}{c}
0 \\
-\sum_{e=1}^{n_{e l m}} \tau_{e}\left\langle\nabla q_{h},\left(\nabla p_{h}-\mathbf{\Pi}_{h}\right)\right\rangle_{\Omega_{e}}
\end{array}\right]
$$

As a result of the above procedure, the stabilized system of equations proposed by the authors to solve the problem of incompressibility in case of elasto-plastic behavior, is the following:

$$
\begin{aligned}
\left\langle\nabla^{s} \mathbf{v}_{h}, \mathbf{s}_{h}\right\rangle+\left\langle\nabla \cdot \mathbf{v}_{h}, p_{h}\right\rangle-\left\langle\mathbf{v}_{h}, \mathbf{f}\right\rangle-\left\langle\mathbf{v}_{h}, \overline{\mathbf{t}}\right\rangle_{\partial \Omega_{t}} & =0 \\
\left\langle q_{h}, \nabla \cdot \mathbf{u}_{h}\right\rangle-\left\langle q_{h}, \frac{1}{K} p_{h}\right\rangle-\sum_{e=1}^{n_{e l m}} \tau_{e}\left\langle\nabla q_{h} \cdot\left[\nabla p_{h}-\boldsymbol{\Pi}_{h}\right]\right\rangle_{\Omega_{e}} & =0 \\
\left\langle\nabla p_{h}, \boldsymbol{\eta}_{h}\right\rangle-\left\langle\boldsymbol{\Pi}_{h}, \boldsymbol{\eta}_{h}\right\rangle & =0
\end{aligned}
$$

It is important to point out that under these hypotheses the stabilization term (48) does not affect the momentum equation (49), which can be solved as in a standard mixed finite element formulation. On the other hand, the incompressibility equation (50) is stabilized element by element, using a term that depends on the difference between the continuous (projected) and the discontinuous (elemental) pressure gradient. This means that the finer is the mesh, smaller is the contribution to the stabilization term to be added to this equation to make possible a stable solution. Finally, it must be observed that in this formulation a third nodal variable $\boldsymbol{\Pi}_{h}$ has to be solved. However, in the next section we will show that it is possible to overcome this drawback and achieve a robust and efficient solution.

\section{Implementation aspects}

Many alternatives can be considered to solve the proposed system of equations (49)-(51). The strategy preferred by the authors consists of a simultaneous solution at time $t=t^{(n+1)}$ of the balance of momentum together with the pressure equation. The projection of the pressure gradient $\boldsymbol{\Pi}_{h}$ obtained at time $t=t^{(n)}$ is used during the equilibrium iterations of the next time 


\section{Box-2: Solution algorithm}

1) Time step $n+1$ :

Initialize iteration $i=0 \rightarrow[\mathbf{U}, \mathbf{P}]^{T(n+1,0)}=[\mathbf{U}, \mathbf{P}]^{T(n)}$

2) For the $(i+1)^{\text {th }}$ iteration:

Compute $\triangle \mathbf{U}^{(n+1, i+1)}$ and $\triangle \mathbf{P}^{(n+1, i+1)}$ as:

$$
\left[\begin{array}{cc}
\mathbf{K}_{\mathrm{dev}}^{(n+1, i)} & \mathbf{G} \\
\mathbf{G}^{T} & -\frac{1}{K} \mathbf{M}_{p}-\mathbf{L}
\end{array}\right]\left[\begin{array}{c}
\triangle \mathbf{U} \\
\triangle \mathbf{P}
\end{array}\right]^{(n+1, i+1)}=-\left[\begin{array}{l}
\mathbf{R}_{1} \\
\mathbf{R}_{2}
\end{array}\right]^{(n+1, i)}
$$

3) Update variables:

$[\mathbf{U}, \mathbf{P}]^{T(n+1, i+1)}=[\mathbf{U}, \mathbf{P}]^{T(n+1, i)}+[\triangle \mathbf{U}, \triangle \mathbf{P}]^{T(n+1, i+1)}$

4) Check convergenge

5) If not converged: new iteration: $i \leftarrow i+1 \quad$ (go to 2 )

6) If converged: set: $[\mathbf{U}, \mathbf{P}]^{T(n+1)}=[\mathbf{U}, \mathbf{P}]^{T(n+1, i+1)}$

7) Compute $\boldsymbol{\Pi}^{(n+1)}$ :

$$
\boldsymbol{\Pi}^{(n+1)}=\mathbf{M}^{-1} \mathbf{G} \mathbf{P}^{(n+1)}
$$

8) Next time sep: $n \leftarrow n+1 \quad$ (go to 1$)$ 
step. This strategy has proved to be effective without loss of precision nor robustness. A brief summary of the algorithm and matrix format implemented is presented in Box-2 (more details can be found in Chiumenti et al. (2002)).

Expressions $\mathbf{R}_{1}^{n+1}$ and $\mathbf{R}_{2}^{n+1}$ in Box- 2 are the residuals vectors associated to equations (49) and (50), respectively. Moreover, the elemental submatrices corresponding to local nodes $A$ and $B$ that must be assembled for the solution of the global system of equations are the following:

$$
\begin{aligned}
{\left[\mathbf{K}_{\mathrm{dev}}^{A B}\right]^{(e)} } & =\left[\int_{\Omega^{e}} \mathbf{B}_{A}^{T} \mathbf{D}_{\mathrm{dev}} \mathbf{B}_{B} d \Omega\right]^{(e)} \\
{\left[\mathbf{G}^{A B}\right]^{(e)} } & =\left[\int_{\Omega^{e}}\left[\mathbf{b}_{A}\right] N_{p}^{B} d \Omega\right]^{(e)} ; \quad\left[\mathbf{b}_{A}\right]=\left[N_{, x}^{A} N_{, y}^{A} N_{, z}^{A}\right]^{T} \\
{\left[L^{A B}\right]^{(e)} } & =\left[\tau_{e} \int_{\Omega^{e}}\left[\mathbf{b}_{A}\right]^{T}\left[\mathbf{b}_{B}\right] d \Omega\right]^{(e)} \\
{\left[M_{p}^{A B}\right]^{(e)} } & =\left[\int_{\Omega^{e}} N^{A} N^{B} d \Omega\right]^{(e)}
\end{aligned}
$$

where $\mathbf{D}_{\text {dev }}$ is the deviatoric part of the constitutive tensor; $\mathbf{G}$ and $\mathbf{G}^{T}$ are the gradient and the divergence operators, respectively; $\mathbf{L}$ is the Laplacian operator and $\mathbf{M}$ y $\mathbf{M}_{p}$ are the "mass" matrices associated to the displacement and pressure fields, respectively.

Finally, note that using a lumped projection operator $\overline{\mathbf{M}}$ it is possible to transform equation 7) in Box-2 into a trivial vector operation leading to a really efficient solution algorithm.

\section{Numerical results}

The formulation presented in the preceding sections is illustrated below in a number of numerical simulations. The objective is to show the performance of the proposed algorithm when a J2-elasto-plastic constitutive behavior is considered. Incompressibility condition are assumed in all the benchmark proposed. Performance of the method is tested considering either 2D planestrain triangular meshes or 3D tetrahedral meshes. To show the behavior of the element under extreme situations, coarse meshes are used in all the tests. Newton-Raphson method combined with a line search optimization procedure, is used to solve the non-linear system of equations arising from 
the spatial and temporal discretization of the weak form of the stabilized problem. Calculations are performed with an enhanced version of the finite element code COMET (see Cervera et al. (2002)) developed by the authors at the International Center for Numerical Methods in Engineering (CIMNE).

\subsection{D Tension benchmark}

The benchmark proposed consists of a square specimen $\left(10 \times 10 \mathrm{~cm}^{2}\right)$ in plane-strain conditions. Two rigid plates have been fixed to the top and the bottom of the specimen. The loading condition consists of a prescribed displacement of the top plate of $1 \mathrm{~mm}$ in the vertical direction. The bottom plate is fixed. Young's modulus is $E=2.0 \times 10^{5} \mathrm{MPa}$ and the incompressibility condition is enforced by setting Poisson's ratio to $\nu=0.4999$. A perfect plasticity model is assumed considering an elastic limit of $\sigma_{o}=150 \mathrm{MPa}$. The proposed formulation T1P1 is compared to the solution of the mixed mean dilatation/pressure quadrilateral element, generally referred to as Q1P0 element (Simo et al. (1985)) and the standard Galerkin formulation for triangular elements $\mathrm{T} 1$.

As a first result, observe in figure 1 that pressure contourfills present similarity for the proposed T1P1 and Q1P0 formulations, even if coarse meshes are used. Locking effects induced by the standard Galerkin formulation can be clearly observed in the same figure. Figure 2 shows the equivalent plastic strain contours obtained with the different formulations. Also in this case there is good agreement between the proposed and the Q1P0 formulations. Observe that plastic deformations for the standard Galerkin formulation are localizing incorrectly along a fictitious shear band induced by the finite element mesh. The same phenomenon has been observed in other problems, leading to the conclusion that the proposed formulation is able to produce results that do not depend on the orientation of the mesh used.

Figure 3-a shows the global response of the specimen in terms of vertical reaction versus vertical displacement. The proposed mixed stabilized formulation compares very well with the results achieved using the Q1P0 formulation. The standard formulation shows a very clear locking effect both in the elastic and in the plastic regimes, with incorrect load-displacement slopes in both situations. Finally, figure 3-b compares the same plots in case of compressible elastic behaviour (Poisson's ratio $\nu=0.3$ ). It is possible to appreciate how the formulation proposed by the authors is still valid in this case. More examples showing the performance of the formulation in 


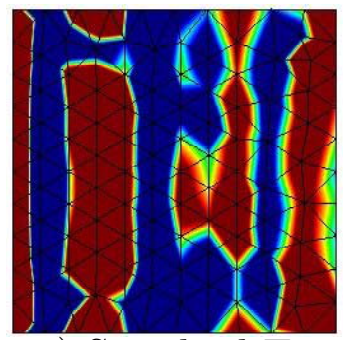

a) Standard T1

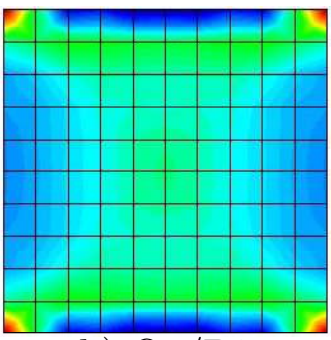

b) $\mathrm{Q} 1 / \mathrm{P} 0$

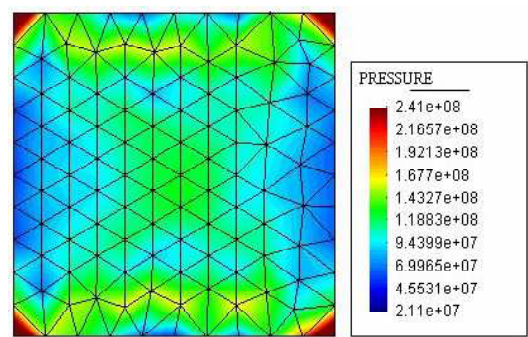

c) Proposed T1P1

Figure 1: Comparison of the pressure field among the different formulations

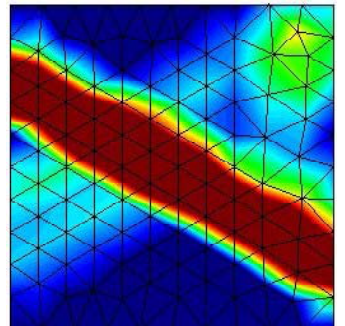

a) Standard T1

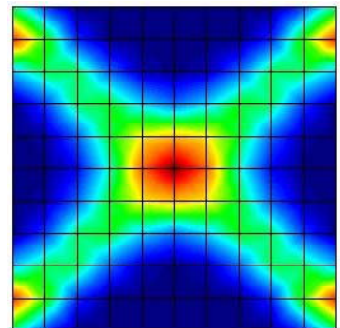

b) $\mathrm{Q} 1 / \mathrm{P} 0$

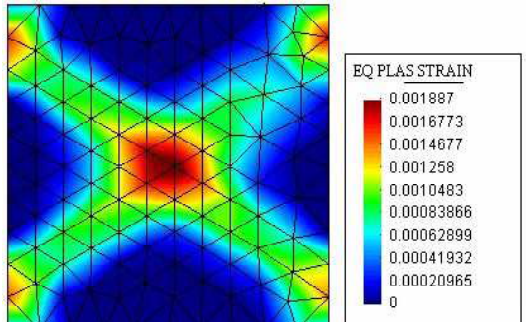

c) Proposed T1P1

Figure 2: Comparison of the equivalent plastic strain field among the different formulations 
compressible and incompressible elasticity can be found in Chiumenti et al. (2002).

\subsection{Plane strain Cook's membrane problem}

The Cook's membrane problem is a bending dominated example that has been used by many authors as a reference test to check their element formulations, see Simo et al. (1990), Miehe (1994) among others. Here it will be used to compare results for incompressible J2-plasticity, showing the behavior of the algorithm for triangular elements. As a reference solution, the mixed mean dilatation/pressure quadrilateral element, generally referred to as Q1P0 (Simo et al. (1985)), is used. The problem consists of a tapered panel, clamped on one side and subjected to a shearing load at the free end. In order to test the convergence behavior of the different formulations, the problem has been discretized into $2 \times 2,5 \times 5,10 \times 10,20 \times 20$ and 50x50 triangular and quadrilateral finite element meshes. Young's modulus is $E=70$, the elastic limit is $\sigma_{o}=0.243$, linear isotropic and kinematic hardening are $h=0.135$ and $K_{h}=0.015$, respectively, and the incompressibility condition is enforced by setting Poisson's ratio to $\nu=0.4999$.

In Figure 4 the comparison among different formulations in case of incompressible plasticity is shown. Q1P0 mixed mean dilatation/pressure approach, T1 standard elements and the proposed mixed formulation for triangular elements are compared. The same figure shows how the proposed formulation converges to the exact solution faster than the Q1P0 mixed approach, even if triangular meshes are used. The figure also shows the poor performance of $\mathrm{T} 1$ standard elements within the context of nearly incompressible plasticity, due to an extreme locking effect. Figure 5 shows the pressure contour fills obtained with the three formulations, using the finest 50x50 quadrilateral (Q1P0) and triangular (T1 and T1P1 stabilized) meshes. It is possible to observe the similarity between the Q1P0 pressure field and the one obtained using the formulation proposed by the authors and on the other hand, the terrible locking effects of a standard Galerkin formulation.

\subsection{D Compression test}

The objective of this problem is to show the performance of the proposed formulation when a tetrahedral mesh is used to solve a $3 \mathrm{D}$ problem. It has been observed that the sensitivity of enhanced element formulations causes 


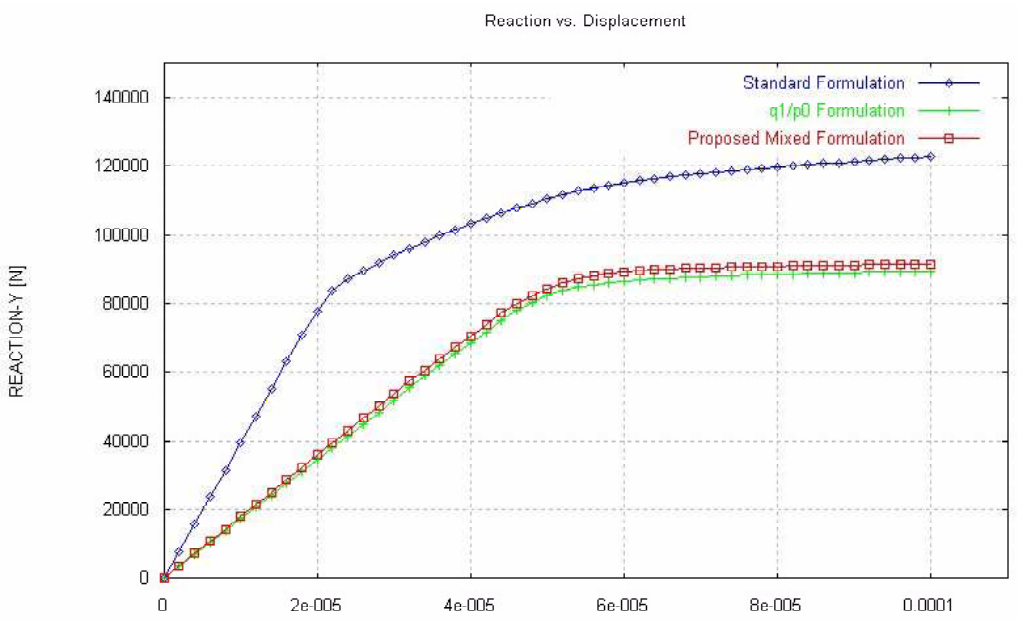

a) Incompressible elasticity and J2-plastic model

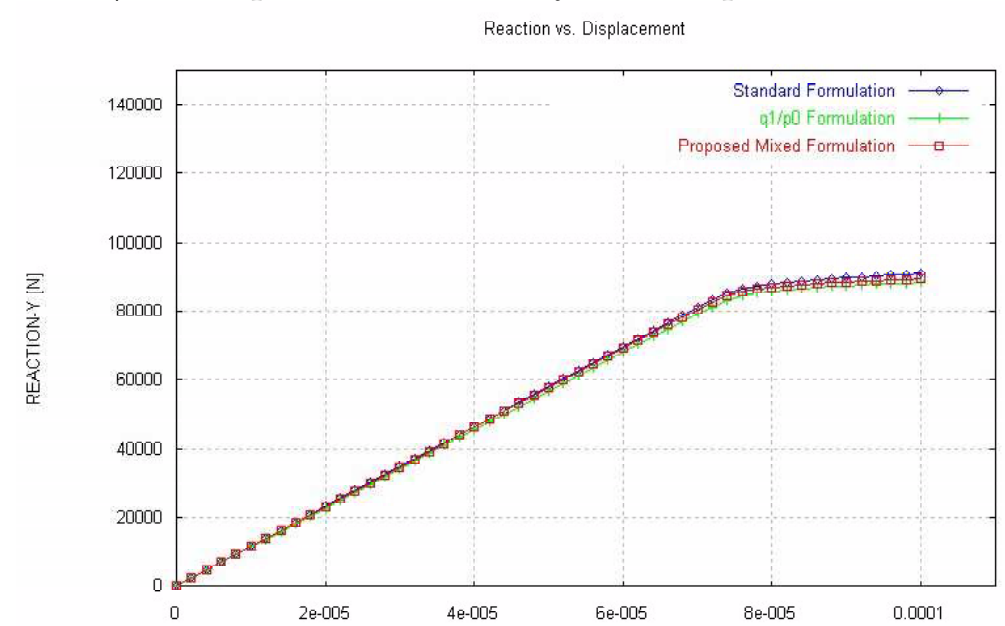

b) Compressible elasticity and J2-plastic model

Figure 3: Vertical reaction vs. vertical displacement of the top plate. 
COOK'S MEMBRANE PROBLENः Elasto-plastic analysis, INCOMPRESSIBLE case (Poisson's ratio $=0.4999$ ) Top Corner Displacement [mm]

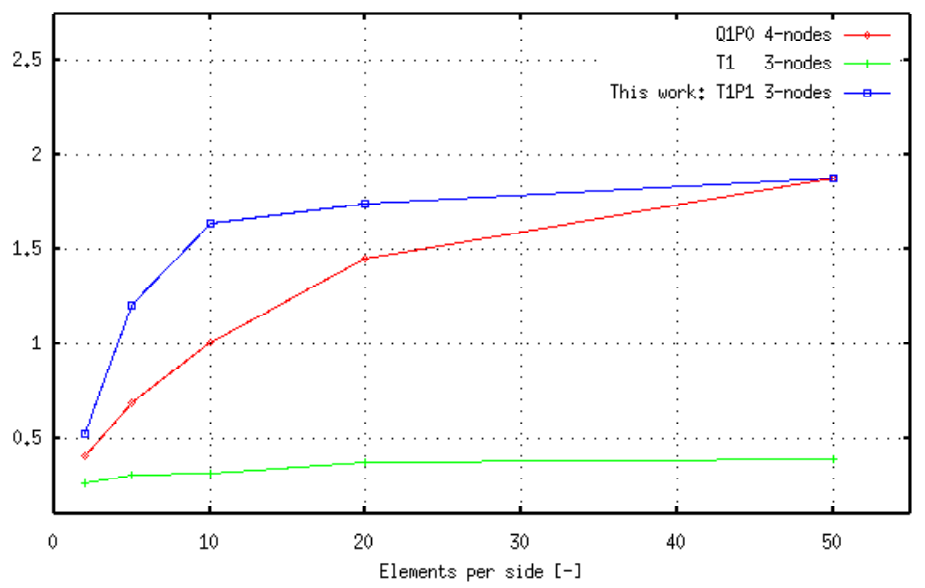

Figure 4: Plane strain Cook's membrane problem: convergence of different element formulations for quasi-incompressible J2-plasticity. T1: standard displacement model for triangular elements, Q1P0: mixed mean dilatation/pressure approach for quadrilateral elements and T1P1: proposed mixed formulation for triangular elements. 

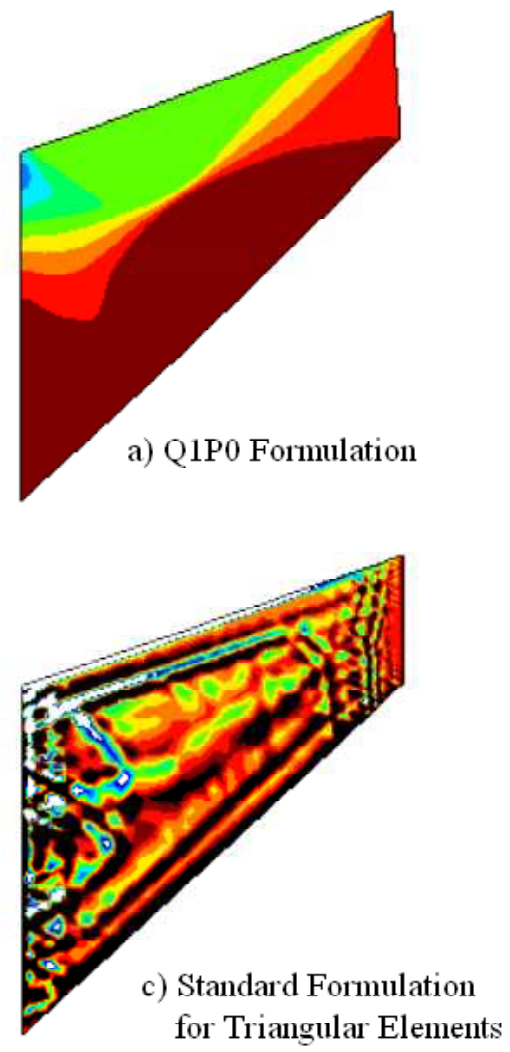
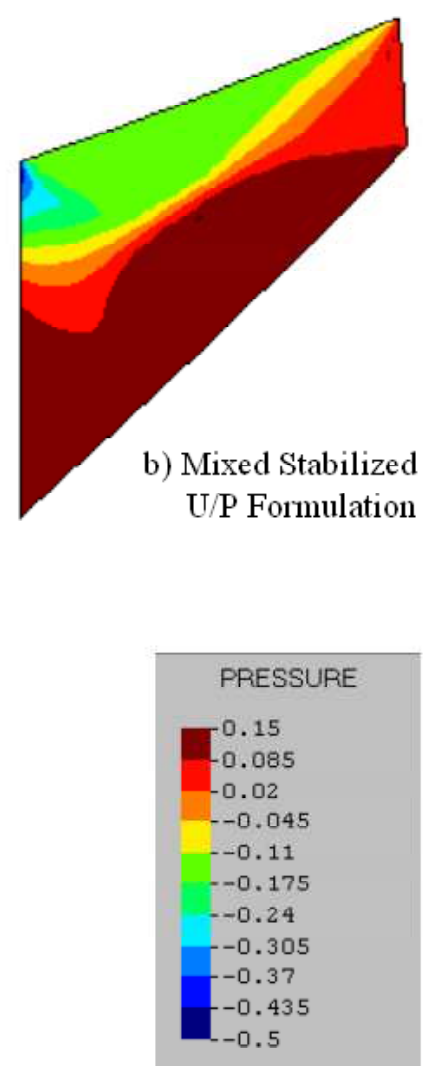

Figure 5: Plane strain Cook's membrane problem: pressure contour fill for quasi-incompressible J2-plasticity. Q1P0: mixed mean dilatation/pressure approach for quadrilateral elements, T1P1: proposed mixed formulation for triangular elements and T1: standard displacement model for triangular elements. 


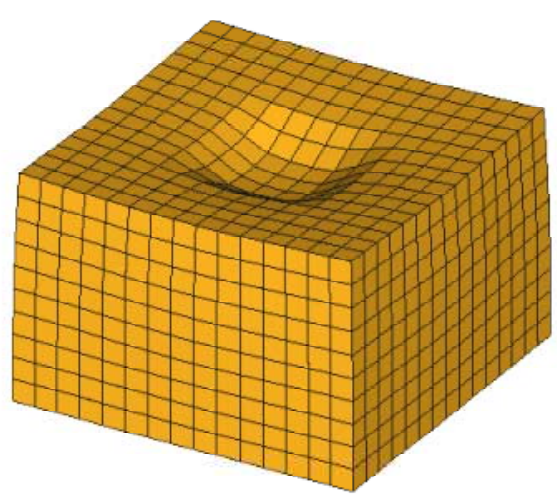

a) Q1/P0 Formulation

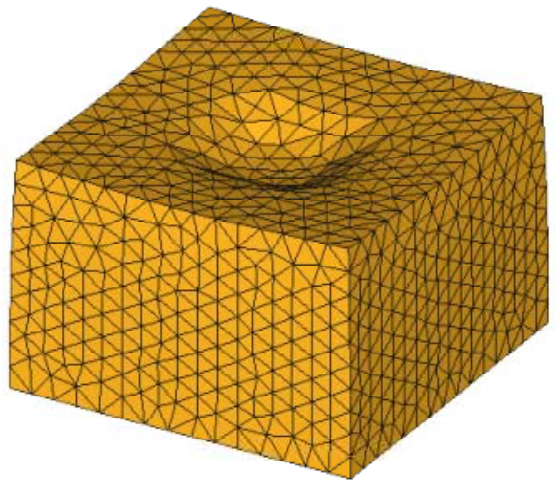

b) Mixed Stabilized U/P Formulation

Figure 6: Deformed meshes: a) Hexahedral mesh used together with the Q1P0 formulation; b) Tetrahedral mesh used for the proposed mixed stabilized $\mathrm{U} / \mathrm{P}$ formulation

difficulties in compression problems, due to numerical instabilities referred to as hourglassing effect. The performance of the proposed formulation has been checked also in that situation. A $0.3 \times 0.3 \times 0.2 \mathrm{~m}^{3}$ block is incrementally subjected to a compression load $(F=1.0 E 4 \quad M P a)$ applied on its upper surface (see Figure 6). Elasto-plastic constitutive behavior has been assumed (Young's modulus: $E=2.0 \times 10^{5} \mathrm{MPa}$; elastic limit: $\sigma_{o}=150 \mathrm{MPa}$ ) together with incompressibility condition (Poisson's ratio: $\nu=0.4999$ ). Results obtained with the Q1P0 formulation using a 15x15x10 hexahedral mesh

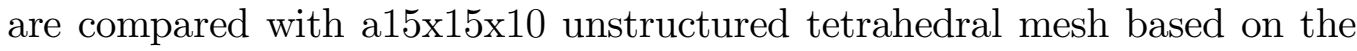
proposed formulation. Figure 6 shows the deformed shapes obtained mesh while Figure 7 compares the pressure contour fill being this variable the most sensitive to the any stabilization technique adopted. It is possible to appreciate in both figure the similarity in terms of deformability and stress response for comparable finite element discretizations. 


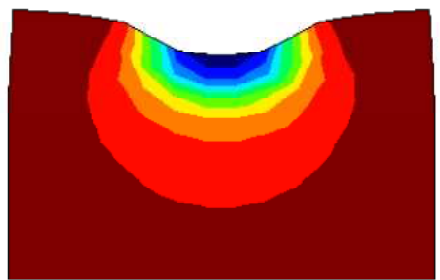

a) Q1/P0 Formulation

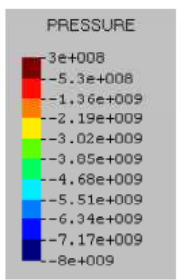

-

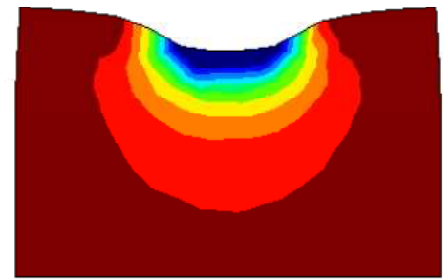

b) Mixed Stabilized U/P Formulation

Figure 7: Pressure contourfill in a central section: a) Q1P0 formulation; b) Mixed stabilixed $\mathrm{U} / \mathrm{P}$ formulation

\section{Conclusions}

In this paper a stabilized finite element method to deal with incompressibility in solid mechanics problems is presented. The method is based on the orthogonal sub-grid scales approach and circumvents the Babuška-Brezzi condition, allowing an accurate and robust formulation suitable for linear triangular and tetrahedral elements. The formulation, presented in a previous work for incompressible elasticity (Chiumenti et al. (2002)) is extended here to the context of incompressible J2-plasticity. A staggered algorithm together with an explicit solution of the projection of the pressure gradient lead to a competitive procedure suitable for industrial applications. Extension of this technology to the context of large-strain elasto-plasticity will be published soon.

\section{Acknowledgments}

The authors are thankful for the financial support of the Spanish Ministerio de Ciencia y Tecnología within the project 2FD1997-0512-CO2-02. Useful discussions with Prof. R. Codina are gratefully acknowledged.

\section{References}

[1] Agelet de Saracibar, C., Cervera, M. and Chiumenti, M., 2001. On 
the constitutive modelling of coupled thermo-mechanical phase-change problems, International Journal of Plasticity, 17, 1565-1622.

[2] Brezzi, F. and Fortin, M., 1991. Mixed and Hybrid Finite Element Methods, Spinger, New York.

[3] Cervera, M., Agelet de Saracibar, C. and Chiumenti, M., 2002. COMET: Coupled Mechanical and Thermal Analysis. Data Input Manual, Version 5.0, Technical report IT-308, htpp://www.cimne.upc.es.

[4] Chiumenti, M., Valverde, Q., Agelet de Saracibar, C. and Cervera, M., 2002. A stabilized formulation for elasticity using linear displacement and pressure interpolations, Comp. Meth. in Appl. Mech. and Eng. 191, 5253-5264.

[5] Codina, R., 2000. Stabilization of incompressibility and convection through orthogonal sub-scales in finite element methods, Comp. Meth. in Appl. Mech. and Eng. 190, 1579-1599.

[6] Codina, R., 2002. Stabilized finite element approximation of transient incompressible flows using orthogonal subscales, Comp. Meth. in Appl. Mech. and Eng. 191, 4295-4321.

[7] Codina, R. and Blasco, J., 2000. Stabilized finite element method for transient Navier-Stokes equations based on pressure gradient projection, Comp. Meth. in Appl. Mech. and Eng. 182, 287-300.

[8] Garikipati, K. and Hughes, T.J.R., 1998. A study of strain localization in a mulitiple scale framework -the one dimensional problem, Comp. Meth. in Appl. Mech. and Eng. 159, 193-222.

[9] Garikipati, K. and Hughes, T.J.R., 2000. A variational multiscale approach to strain localization - formulation for multidimensional problem, Comp. Meth. in Appl. Mech. and Eng. 188, 39-60.

[10] Hughes, T.J.R., 1987. The finite element method: linear static and dynamic finite element analysis, Prentice-Hall, Inc. Englewood Cliffs, New Jersey.

[11] Hughes, T.J.R., 1995. Multiscale phenomena: Green's function, Dirichlet-to Neumann formulation, subgrid scale models, bubbles and 
the origins of stabilized formulations, Comp. Meth. in Appl. Mech. and Eng. 127, 387-401.

[12] Klass, O., Maniatty, A. and Shephard, M.S., 1999a. A stabilized mixed finite element method for finite elasticity. Formulation for linear displacement and pressure interpolation, Comp. Meth. in Appl. Mech. and Eng. 180, 65-79.

[13] Klass, O., Maniatty, A. and Shephard, M.S., 1999b. Three dimensional stabilized finite element model for finite deformation process. Proceedings 7th International Symposium on Plasticity and its Current applications, Cancun, Mexico.

[14] Miehe, C., 1994. Aspects of the formulation and finite element implementation of large strain isotropic elasticity, Int. Jour. for Num Methods in Eng. 37, 1981-2004.

[15] Oñate, E., Rojek, J., Taylor, R.L. and Zienkiewicz, O.C., 2002. Finite calculus formulation for incompressible solids using linear triangles and tetrahedra, Submitted to Int. Jour. for Numerical Meth. in Eng.

[16] Simo, J.C. and Rifai, M.S., 1990. A class of mixed assumed strain methods and the method of incompatible modes, Int. Jour. for Num Methods in Eng. 29, 1595-1638.

[17] Simo, J.C., Taylor, R.L. and Pister, K.S., 1985. Variational and projection methods for the volume constraint in finite deformation elastoplasticity, Comp. Meth. in Appl. Mech. and Eng. 51, 177-208

[18] Taylor, R.L., 1999. A mixed formulation for triangular and tetrahedral finite elements, In Conference proceedings, Metodos Numericos en la Ingenieria, SEMNI, Barcelona, Spain.

[19] Zienkiewicz, O.C. and Taylor, R.L., 1994. Finite element method, Volume-1, 4th ed., McGraw-Hill.

[20] Zienkiewicz, O.C., Rojek, J., Taylor, R.L. and Pastor, M., 1998. Triangles and tetrahedra in explicit dynamic codes for solids, Int. Jour. for Num Methods in Eng. 43, 565-583 